\title{
Recurrent cholangiocarcinoma with long-term survival by multimodal treatment: A case report
}

\author{
YUKI SOTA $^{1}$, TAKAHIRO EINAMA ${ }^{1}$, KAZUKI KOBAYASHI ${ }^{1}$, IBUKI FUJINUMA ${ }^{1}$, TAKAZUMI TSUNENARI ${ }^{1}$, \\ YASUHIRO TAKIHATA ${ }^{1}$, TOSHIMITSU IWASAKI ${ }^{1}$, YOICHI MIYATA $^{1}$, KOICHI OKAMOTO $^{1}$, \\ YOSHIKI KAJIWARA ${ }^{1}$, EIJI SHINTO ${ }^{1}$, HIRONORI TSUJIMOTO ${ }^{1}$, SHIGEO YASUDA ${ }^{2}$, YUKA \\ ISOZAKI $^{2}$, SHIGERU YAMADA $^{2}$, JUNJI YAMAMOTO ${ }^{1}$, HIDEKI UENO $^{1}$ and YOJI KISHI ${ }^{1}$ \\ ${ }^{1}$ Department of Surgery, National Defense Medical College, Tokorozawa, Saitama 359-8513; \\ ${ }^{2}$ Department of Gastrointestinal Oncology, QST Hospital International Therapy Research Center, \\ Chiba 263-8555, Japan
}

Received June 8, 2020; Accepted December 9, 2020

DOI: $10.3892 / \mathrm{mco} .2021 .2234$

\begin{abstract}
Long-term outcomes after surgical resection of bile duct cancer remain unsatisfactory, and survival, particularly after tumor recurrence, is poor. Gemcitabine and cisplatin combination (GC) therapy is the standard first-line treatment; however, second-line approaches are yet to be established. Radiotherapy may prolong the survival of patients with advanced biliary tract cancer, and particle radiotherapy delivers a more concentrated dose than conventional radiotherapy to deeper tumors. The present report describes the long-term survival of a 65 -year-old man with distal bile duct cancer of pathological stage IIA (T2N0M0; depth of invasion, $5.5 \mathrm{~mm}$ ) following multimodal treatment. Following subtotal stomach-preserving pancreatoduodenectomy, multiple hepatic recurrences were identified 9 months later, and GC therapy was initiated. The tumors were no longer evident 18 months later, and GC therapy was discontinued at the patient's request. A computed tomography (CT) scan performed 30 months after surgery identified a new solitary hepatic recurrence and duke pancreatic monoclonal antigen type-2 (DUPAN-2) levels were increased. Further GC therapy was declined. Carbon ion radiotherapy (CIRT) at a dose of 60 Gy [relative
\end{abstract}

Correspondence to: Dr Takahiro Einama, Department of Surgery, National Defense Medical College, 3-2 Namiki, Tokorozawa, Saitama 359-8513, Japan

E-mail: einama0722@ndmc.ac.jp

Abbreviations: CIRT, carbon ion radiotherapy; CT, computed tomography; DUPAN-2, duke pancreatic monoclonal antigen type-2; CA19-9, carbohydrate antigen 19-9; FDG-PET/CT, fluorodeoxyglucosepositron emission tomography/computed tomography; GC, gemcitabine and cisplatin; PBT, proton beam therapy; RBE, relative biological effectiveness; GPT, gemcitabine-concurrent proton radiotherapy; NIRS, National Institute of Radiological Science

Key words: cholangiocarcinoma, hepatic recurrence, carbon ion radiotherapy, gemcitabine, cisplatin, chemotherapy biological effectiveness (RBE)-weighted absorbed dose] was then delivered in four fractions over 4 days [15 Gy (RBE)/day]. Tumor size decreased on CT, and fluorodeoxyglucose-positron emission tomography/CT revealed a decline in the standardized uptake value of the tumor after 2 months, with decreased DUPAN-2 levels. Following regrowth of the hepatic recurrence, CIRT was repeated at a dose of $66 \mathrm{~Gy}(\mathrm{RBE})$ in four fractions over 4 days [16.5 Gy (RBE)/day] and stable disease was maintained for 19 months. After 19 months, CT revealed tumor regrowth and another new metastatic lesion was identified in the left kidney. The patient received systematic chemotherapy again and died of the disease 81 months after the initial surgery. In conclusion, CIRT is a potential treatment option to control solitary recurrence of biliary tract cancer.

\section{Introduction}

Generally, surgical resection is the only potentially curative treatment for patients with cholangiocarcinoma. However, tumor recurrence occurs in $\sim 70 \%$ of patients with biliary tract cancer, even after curative resection, and 5-year postoperative survival is less than $50 \%$ (1-3). Long-term survival can rarely be expected once tumor recurrence occurs because the recurrence means 'systematic disease'. Most of the recurrence cases, the curative resection is impossible and the systematic chemotherapy is selected.

Gemcitabine and cisplatin (GC) combination therapy is the first choice of treatment for recurrent or unresectable biliary tract cancer (4), but a strategy for second-line therapies has not yet been established.

Several reports have suggested that conventional or particle radiotherapy might prolong the survival of patients with advanced biliary tract cancer $(5,6)$. It is known that particle therapy has a higher dose concentration compared with conventional radiotherapy and is effective in treating deeply located tumors. Particle therapy is divided into proton beam radiotherapy and heavy particle therapy. Both of them show strong cytotoxicity as a result of high linear energy transfer, unlike the alternative X-ray beam therapy. In heavy particle therapy, carbon ions are usually used. They have potential advantage 


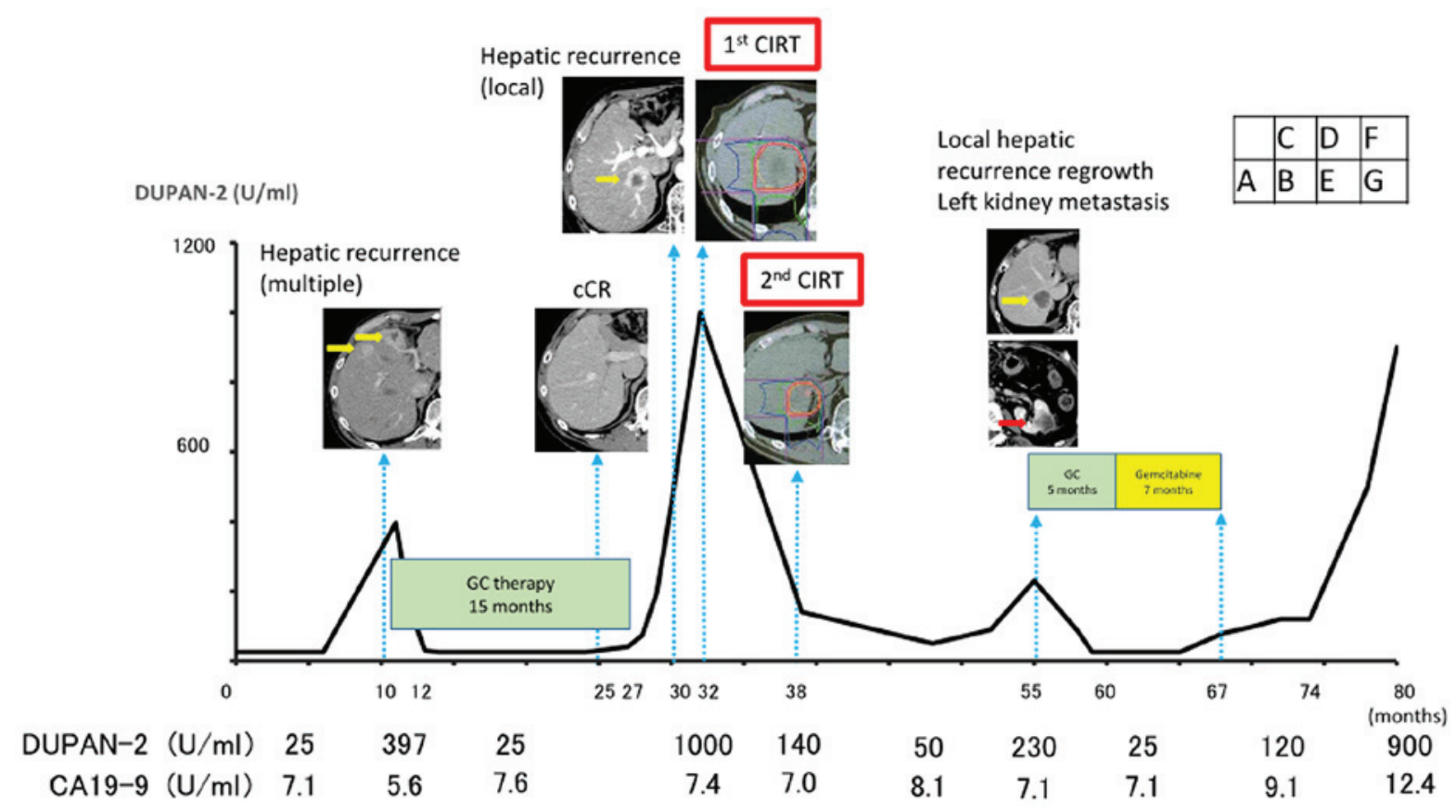

Figure 1. Changes in DUPAN-2 levels following the clinical course and the quantified data of DUPAN-2 and CA19-9. (A) Postoperative CT scan at 10 months revealing multiple solid liver tumors (yellow arrows). The largest tumor was located in segment 4 and had a diameter of $20 \mathrm{~mm}$. (B) CT scan revealing the complete disappearance of the hepatic tumors after GC therapy. (C) A postoperative CT scan at 30 months demonstrated one ring-like enhancement mass measuring $30 \mathrm{~mm}$ in diameter in segment 6 (yellow arrow). Planning CT scans demonstrating (D) first and (E) second CIRT imaging. A postoperative CT scan at 55 months demonstrated (F) the same local hepatic recurrence that had increased in size up to $45 \mathrm{~mm}$ in diameter (yellow arrow) and (G) identified a new metastatic lesion in the left kidney (red arrow). DUPAN-2, duke pancreatic monoclonal antigen type-2; CA19-9, carbohydrate antigen 19-9; GC, gemcitabine and cisplatin; CIRT, carbon ion radiotherapy; cCR, clinical complete response; Lt.

compared to protons. For example, they provide a better physical dose distribution because lateral scattering is more lessened. Furthermore, carbon ions exhibit a higher linear energy transfer than protons. This leads to a higher relative biological effectiveness (RBE), where damage caused by carbon ions is clustered in the DNA, overwhelming the cellular repair system (7). Several cohort studies showed the effectiveness of proton beam therapy for biliary tract cancer (8-11), whereas heavy particle therapy for biliary tract cancer has not been evaluated.

We experienced a case of recurrent distal bile duct cancer that achieved long-term survival with a multimodal treatment strategy of systematic chemotherapy and heavy particle therapy. So, we will report this case with additional literature review.

\section{Case report}

A 65-year-old male underwent a subtotal stomach-preserving pancreatoduodenectomy for distal bile duct cancer. The pathological depth of tumor invasion was $5.5 \mathrm{~mm}$ and there was no lymph node metastasis with a pathological stage of IIA (T2N0M0) according to the eighth Union for International Cancer Control staging (12). The patient was discharged 21 days after surgery without any postoperative complications and did not receive any adjuvant chemotherapy.

Multiple hepatic recurrences were recognized on computed tomography (CT) performed 9 months after the surgery (Fig. 1A), and thus GC therapy was initiated. This therapy consisted of gemcitabine $(1,000 \mathrm{mg}$ per square meter of body-surface area) and cisplatin (25 $\mathrm{mg}$ per square meter of body-surface area) and was performed 10 cycles with $100 \%$ dose. After 18 months, all hepatic tumors had completely disappeared (Fig. 1B). The GC therapy was discontinued at the patient's request.

Three months later, serum DUPAN-2 levels had elevated, and a CT scan revealed a new hepatic recurrence (Fig. 1C). However, serum carbohydrate antigen 19-9 (CA19-9) levels had not elevated. We proposed that GC therapy should be restarted, but the patient declined because he was suffering from peripheral neuropathy. Because no other recurrences were detected by $\mathrm{CT}$, we consulted with radiologists, and particle therapy was recommended. The patient was treated with heavy particle therapy [carbon ion radiotherapy (CIRT)]. The total dose was $60 \mathrm{~Gy}(\mathrm{RBE})$ in four fractions for 4 days [15 Gy (RBE)/day] (Fig. 1D). There were no adverse events during the first CIRT. CT scan revealed shrinkage of the hepatic tumor. In addition, the level of standard uptake value at the center of hepatic recurrence decreased from 8.1 to 0 by fluorodeoxyglucose-positron emission tomography/computed tomography (FDG-PET/CT). Serum DUPAN-2 levels also decreased.

Six months after the first CIRT, a hepatic tumor was identified at the same site upon CT. The patient received CIRT again, with a total dose of $66 \mathrm{~Gy}$ (RBE) in four fractions for 4 days [16.5 Gy (RBE)/day] (Fig. 1E). The only adverse event was slight dermatitis. After the second CIRT, the serum DUPAN-2 level decreased to within the normal range.

Nineteen months after the second CIRT, CT showed a hepatic tumor regrowth with a diameter of $45 \mathrm{~mm}$ (Fig. 1F). FDG-PET/CT confirmed an elevated standardized uptake value from 0 to 7.4 in this lesion. Furthermore, a new metastatic lesion was recognized in the left kidney (Fig. 1G). We started GC therapy again because it had been effective against the initial recurrence, and the time interval since the first treatment was approximately 
Table I. Previous reports regarding the clinical outcomes of PBT for biliary tract cancer.

\begin{tabular}{|c|c|c|c|c|c|c|}
\hline Author, year & $\begin{array}{l}\text { Number } \\
\text { of patients }\end{array}$ & Tumor type and characteristics & $\begin{array}{l}\text { PBT dose, } \\
\text { fractionation, } \\
\text { technique }\end{array}$ & $\begin{array}{l}\text { Median } \\
\text { follow-up, } \\
\text { months }\end{array}$ & Survival outcomes & (Refs.) \\
\hline $\begin{array}{l}\text { Ohkawa et al, } \\
2015\end{array}$ & 14 & $\begin{array}{l}\text { Intrahepatic cholangiocarcinoma. } \\
\text { Stage II }(1 / 14 ; 7 \%) ; \text { stage IIIA } \\
(4 / 14 ; 29 \%) ; \text { stage IIIC }(5 / 14 ; \\
36 \%) ; \text { stage IV }(4 / 14 ; 29 \%)\end{array}$ & $\begin{array}{l}\text { Median } 72.6 \\
\text { CGE in } 26 \\
\text { fractions }\end{array}$ & 12 & $\begin{array}{l}1 \text { y OS } 50 \% ; 1 \text { y PFS } \\
36 \%, \text { LP in } 6 / 14(43 \%) ; \\
\text { LR in } 2 / 14(14 \%) ; \\
\text { Out-of-field recurrence } \\
\text { in } 7 / 14(50 \%) ; \text { DM } \\
\text { in } 4 / 14(28 \%)\end{array}$ & (8) \\
\hline $\begin{array}{l}\text { Makita et al, } \\
2014\end{array}$ & 28 & $\begin{array}{l}\text { Cholangiocarcinoma. Intrahepatic } \\
(6 / 28 ; 21 \%) ; \text { hilar }(6 / 28 ; 21 \%) ; \\
\text { distal extrahepatic }(3 / 28 ; 11 \%) ; \\
\text { gallbladder }(3 / 28 ; 11 \%) ; \\
\text { local/nodal recurrence } \\
(10 / 28 ; 36 \%)\end{array}$ & $\begin{array}{l}\text { Median } 68.2 \\
\text { CGE in } 31 \\
\text { fractions }\end{array}$ & 12 & $\begin{array}{l}1 \text { y LC } 68 \%, 1 \text { y } \\
\text { PFS } 30 \% .1 \text { y OS } \\
49 \% . \text { Increased LC } \\
\text { with BED>70 Gy } \\
(\mathrm{P}=0.002)\end{array}$ & (9) \\
\hline
\end{tabular}

PBT, proton beam radiotherapy; CGE, cobalt gray equivalent; OS, overall survival; LP, local progression; DM, distant metastasis; PFS, progression-free-survival.

35 months. We continued GC therapy for 5 months, and then switched to gemcitabine monotherapy because of the peripheral neuropathy and general malaise. Gemcitabine monotherapy (1,000 mg per square meter of body-surface area) was continued administer 3-weeks on/1-week off for 7 months. The patient died 16 months after cessation of the second chemotherapy because of disease progression. The total survival time after the surgery was 81 months.

\section{Discussion}

We experienced a case of extrahepatic bile duct cancer in which long-term survival was achieved with multidisciplinary treatment including systemic chemotherapy and CIRT after tumor recurrence. The patient survived more than 70 months after the first hepatic recurrence. Although the appearance of a solitary recurrence is rare after complete remission of multiple recurrences following systematic chemotherapy, our case suggested that multiple sessions of CIRT might be able to control the disease and contribute to prolonging overall survival.

To date, no treatment other than GC therapy has been established for unresectable or recurrent biliary tract cancer. Radiation therapy or chemoradiotherapy is reserved for patients who present locally advanced unresectable disease but there is insufficient evidence for its efficacy as a treatment for patients with distant metastasis (13).

Recently, several case reports demonstrated the outcomes of proton beam therapy (PBT) and CIRT for various malignant primary or recurrent tumor types (14-18).

For example, in the research of performing gemcitabineconcurrent proton radiotherapy (GPT) to pancreatic cancer cases, 50 patients were divided into three groups by every protocol of PBT. The median follow-up period was 12.5 months. The scheduled GPT was feasible for all except 6 patients $(12 \%)$ due to acute hematologic. Grade 3 or greater late gastric ulcer and hemorrhage were seen in 5 patients (10\%). The one-year freedom from local-progression, progression-free, and overall survival rates were $74 \%$ (14). Like as this research, PBT provide useful local-regional treatment for hepatocellular carcinoma. In this research, 76 patients were treated and followed prospectively for treatment outcomes. Eleven patients had multiple tumors and $46 \%$ were within the Milan criteria. Patients received 63 Gy delivered over a 3-week period with PBT. All cases completed the full course of treatment. Finally, median progression-free survival for the entire group was 36 months, with a 60\% 3-year progression-free survival rate for patients within the Milan criteria. 18 patients subsequently underwent liver transplantation; $6(33 \%)$ explants showed pathological complete response and $7(39 \%)$ showed only microscopic residual (15). The outcome of performing CIRT for hepatocellular carcinoma is also good. In the 69 cases at National Institute of Radiological Science (NIRS), the five-year local control rate was $81 \%$ and survival rate was $33 \%$. Like with the hepatocellular carcinoma, CIRT for pancreatic cancer is effective. In the cases of locally advanced unresectable pancreatic cancer, the combination of gemcitabine and CIRT improved the outcomes. Second-year local control rate was $58 \%$ and second-year overall survival was $54 \%$ in the 47 cases at NIRS. This result is better than those reported worldwide after surgery alone (7).

CIRT was initiated by the Heavy Ion Medical Accelerator in Chiba, Japan, in 1993. It offers superior dose conformity in the treatment of deeply located malignant tumors compared with conventional X-ray therapy (18). Additionally, its biological effectiveness (a highly localized deposition of energy) can be used for increasing radiation exposure to tumors while minimizing irradiation to adjacent normal tissues. PBT also has this characteristic, but the lateral fall-off around the target is less steep than that of carbon ion beams (19).

Despite this property for cancer treatment, no reports have presented the benefits of CIRT for biliary tract cancer. There have been few studies of PBT in primary, locally recurrent, or nodal metastatic disease (Table I). 
Regarding indications for radiation therapy for biliary tract cancer, Kazuki et al (20) reported that the best indication for curative beam therapy was locally advanced unresectable hepatic hilar cholangiocarcinoma without distant metastasis. However, long-term outcomes after CIRT have not yet been reported. In our case, CIRT was indicated at the time of second recurrence, and good local control was achieved. Hence, the patient survived for eighty months after surgery.

In conclusion, CIRT may be a treatment option for selected patients with solitary recurrence of biliary tract cancer.

\section{Acknowledgements}

The authors would like to thank Dr H. Nikki March for editing a draft of this manuscript.

\section{Funding}

No funding was received.

\section{Availability of data and materials}

All data generated or analyzed during this study are included in this published article.

\section{Authors' contributions}

KK, IF, TT, YT, TI and YM collected the data and assisted with data analysis. KO, YKa, ES, HT, SYas, YI and SYam contributed to interpretation of data and manuscript preparation. YS analyzed the data and wrote the original draft. TE conceived the study, reviewed and revised the manuscript. JY and YKi made substantial contributions to the conception and design. HU made substantial contributions to analysis and interpretation of data. JY, HU and YKi provided supervision of the manuscript. All authors read and approved the final manuscript.

\section{Ethics approval and consent to participate}

Not applicable.

\section{Patient consent for publication}

The patient provided written informed consent for the publication of their data.

\section{Competing interests}

The authors declare that they have no competing interests.

\section{References}

1. Koyama K, Ebata T, Yokoyama Y, Igami T, Sugawara G, Mizuno T, Yamaguchi J and Nagino M: Recurrence after curativeintent resection of perihilar cholangiocarcinoma: Analysis of a large cohort with a close postoperative follow-up approach. Surgery 163: 732-738, 2018.

2. Cillo U, Fondevila C, Donadon M, Gringeri E, Mocchegiani F, Schlitt HJ, Iizermans JNM, Vivarelli M, Zieniewicz K, Olde Damink SWM and Groot Koerkamp B: Surgery for cholangiocarcinoma. Liver Int 39 (Suppl 1): S143-S155, 2019.
3. Nagino M, Ebata T, Yokoyama Y, Igami T, Sugawara G, Takahashi Y and Nimura T: Evolution of surgical treatment for perihilar cholangiocarcinoma: A single-center 34-year review of 574 consecutive resections. Ann Surg 258: 129-140, 2013.

4. Valle J, Wasan H, Palmer DH, Cunningham D, Anthoney A, Maraveyas A, Madhusudan S, Iveson T, Hughes S, Pereira SP, et al: Cisplatin plus gemcitabine versus gemcitabine for biliary tract cancer. N Engl J Med 362: 1273-1281, 2010.

5. Horgan AM, Amir E, Walter T and Knox JJ: Adjuvant therapy in the treatment of biliary tract cancer: A systematic review and meta-analysis. J Clin Oncol 30: 1934-1940, 2012.

6. Monson JR, Donohue JH, Gunderson LL, Nagorney DM, Bender CE and Wieand HS: Intraoperative radiotherapy for unresectable cholangiocarcinoma-the mayo clinic experience. Surg Oncol 1: 283-290, 1992.

7. Kamada T, Tsujii H, Blakely EA, Debus J, De Neve W, Durante M, Jäkel O, Mayer R, Orecchia R, Pötter R, et al: Carbon ion radiotherapy in Japan: An assessment of 20 years of clinical experience. Lancet Oncol 16: e93-e100, 2015.

8. Ohkawa A, Mizumoto M, Ishikawa H, Abei M, Fukuda K, Hashimoto T, Sakae T, Tsuboi K, Okumura T and Sakurai H: Proton beam therapy for unresectable intrahepatic cholangiocarcinoma. J Gastroenterol Hepatol 30: 957-963, 2015.

9. Makita C, Nakamura T, Takada A, Takayama K, Suzuki M, Ishikawa Y, Azami Y, Kato T, Tsukiyama I, Kikuchiet Y, et al: Clinical outcomes and toxicity of proton beam therapy for advanced cholangiocarcinoma. Radiat Oncol 9: 26, 2014.

10. Hong TS, Wo JY, Yeap BY, Ben-Josef E, McDonnell EI, Blaszkowsky LS, Kwak EL, Allen JN, Clark JW, Goyal L, et al: Multi-institutional phase II study of high-dose hypofractionated proton beam therapy in patients with localized, unresectable hepatocellular carcinoma and intrahepatic cholangiocarcinoma. J Clin Oncol 34: 460-468, 2016.

11. Bush DA, Smith JC, Slater JD, Volk ML, Reeves ME, Cheng J, Grove R and de Vera ME: Randomized clinical trial comparing proton beam radiation therapy with transarterial chemoembolization for hepatocellular carcinoma: Results of an interim analysis. Int J Radiat Oncol Biol Phys 95: 477-482, 2016.

12. Brierley JD, Gospodarowicz MK and Wittekind C (eds): TNM Classification of Malignant Tumors. 8th edition. In: Union for International Cancer Control. Wiley-Blackwell, p272, 2017.

13. de Jong MC, Nathan H, Sotiropoulos GC, Paul A, Alexandrescu S, Marques H, Pulitano C, Barroso E, Clary BM, Aldrighetti L, et al: Intrahepatic cholangiocarcinoma: An international multi-institutional analysis of prognostic factors and lymph node assessment. J Clin Oncol 29: 3140-3145, 2011.

14. Mizumoto M, Sugahara S, Nakayama H, Hashii H, Nakahara A, Terashima T, Okumura T, Tsuboi K, Tokuuye K and Sakurai H: Clinical results of proton-beam therapy for locoregionally advanced esophageal cancer. Strahlenther Onkol 186: 482-488, 2010.

15. Terashima K, Demizu Y, Hashimoto N, Jin D, Mima M, Fujii O, Niwa Y, Takatori K, Kitajima N, Sirakawaet S, et al: A phase I/II study of gemcitabine-concurrent proton radiotherapy for locally advanced pancreatic cancer without distant metastasis. Radiother Oncol 103: 25-31, 2012.

16. Bush DA, Kayali Z, Grove R and Slater JD: The safety and efficacy of high-dose proton beam radiotherapy for hepatocellular carcinoma: A phase 2 prospective trial. Cancer 117: 3053-3059, 2011.

17. Schineider RA, Vitolo V, Albertini F, Koch T, Ares C, Lomax A, Goitein $\mathrm{G}$ and Hug EB: Small bowel toxicity after high dose spot scanning-based proton beam therapy for paraspinal/retroperitoneal neoplasms. Strahlenther Onkol 189: 1020-1025, 2013.

18. Kamata T: Clinical evidence of particle beam therapy (carbon). Int J Clin Oncol 17: 85-88, 2012.

19. Ohno T: Particle radiotherapy with carbon ion beams. EPMA J 4: 9, 2013.

20. Kazuki T: Radiation therapy and particle radiotherapy using proton or carbon-ion beam for biliary duct cancer. Journal of Japan Biliary Association 32: 114-123, 2018. (In Japanese) 